\title{
A Hand-Held Probe for Vibro-Elastography
}

\author{
Hassan Rivaz and Robert Rohling \\ Department of Electrical and Computer Engineering, \\ University of British Columbia, Vancouver, Canada \\ rohling@ece.ubc.ca \\ ww. ece.ubc.ca/ ${ }^{\text {rohling }}$
}

\begin{abstract}
Vibro-elastography is a new medical imaging method that identifies the mechanical properties of tissue by measuring tissue motion in response to a multi-frequency external vibration source. Previous research on vibro-elastography used ultrasound to measure the tissue motion and system identification techniques to identify the tissue properties. This paper describes a hand-held probe with a combined vibration source and ultrasound transducer. The design uses a vibration absorption system to counter-balance the reaction forces from contact with the tissue. Simulations and experiments show a high level of vibration absorption. The first elastograms from the probe are also shown.
\end{abstract}

\section{Introduction}

Elastography creates images that depict local tissue stiffness. Such images can be a valuable tool for the diagnosis of disease. For example, prostate and breast cancer are known to exhibit stiff tumors in surrounding softer tissue. Elastography may compliment manual palpation techniques for early detection of the tumors. Static elastography is a well-known technique that uses a quasi-static compression of tissue and simultaneous imaging with ultrasound [5]. Local tissue strain estimates can be obtained by analysis of the ultrasound images (e.g. speckle tracking by cross-correlation of the radiofrequency (RF) signals). The strain is subsequently related to the tissue stiffness.

Vibro-elastography (VE) is a new imaging technique that uses a dynamic excitation source to create tissue motion over a range of frequencies [8]. Ultrasound images are used to capture the tissue motion at multiple locations and time instants. The method then adapts techniques from system identification to estimate the tissue properties from the ultrasound-based strain measurements. In one version of $\mathrm{VE}$, the tissue is modelled as a set of interconnected massspring-damper elements. The values of the density-stiffness-viscosity are then identified by solving an over-determined set of equations of motion. In another version of $\mathrm{VE}$, the tissue properties are identified directly from transfer functions between local tissue regions. In either case, the VE images can be shown to have better quality (lower standard deviation to mean values) compared to static elastography, mainly due to the inherent averaging in the approach over various levels and rates of tissue strain. Moreover, measurements of viscosity and density are also possible with the dynamic approach. So far, good results 
for stiffness and viscosity measurements have been obtained with fixed or rigidly mounted ultrasound transducers. Widespread clinical acceptance would be aided by the development of a hand-held probe so that a standard ultrasound scanning protocol can be performed. This paper describes a combined ultrasound transducer and vibrator system as the next step in the development of VE.

For a hand-held probe, the reaction forces between the tissue and vibrator act as an external disturbance on the probe assembly and will cause unwanted vibration. These vibrations should be minimized to maintain the comfort of the operator and the accuracy of the VE calculations. A light-weight probe can exhibit a large vibration level because it has a low displacement-force impedance. A heavier probe alleviates some vibration but at the cost of operator fatigue. Therefore, the probe design should use some form of vibration absorption.

Four different methods for reducing the vibration are well known: vibration isolation, passive vibration absorption, semi-active vibration absorption and active vibration absorption. For vibration isolation, a spring-damper combination is placed between the source of excitation force and the system to be isolated (Figure 1a). In this figure, $k_{i}$ is the stiffness and $b_{i}$ is the viscosity of the isolating material and $F_{e}$ is the excitation force. To minimize the transmitted force, the spring and damper values should be selected according to the excitation frequency [7. For passive vibration absorption, an additional mass-spring-damper trio is attached to the primary system (Figure 1b). In this figure, $m_{a}, k_{a}$ and $b_{a}$ are the absorber mass, stiffness and viscosity respectively. Unlike the previous method, passive vibration absorption adds one degree of freedom (DOF) to the system. To minimize the vibration of the primary system $\left(x_{p}\right)$, the mass, spring and damper values should be selected according to the frequency range of vibration of the primary system [3]. Yet these absorbers can be designed for only a narrow band of excitation frequencies. There is a trade-off between the frequency bandwidth and the absorption level: the wider the bandwidth, the lower the absorption. Semi-active vibration absorption follows the same idea as passive absorption, but uses a tunable spring and damper (Figure 1c). Varying the spring and damper values allows the absorber to be tuned to different excitation frequencies 2]. For active vibration absorption, an actuator is added to the mass-spring-damper trio of a passive absorber (Figure 1d). Active vibration absorption differs from semi-active vibration absorption because it needs more control effort to suppress the vibration, requiring an external energy source. Semi-active and active vibration absorbers have the capability of cancelling the vibration over a wider frequency range than the first two methods.

For VE, an excitation over the range of $10-50 \mathrm{~Hz}$ is needed, so only active or semi-active vibration absorption methods are suitable. In practice, VE rapidly sweeps this range, and semi-active vibration absorbers have difficulty responding with sufficiently speed. Moreover, the inherent damping produces sub-optimal resonance properties at the frequency of excitation, offering low levels of absorption, especially in the miniature hand-held device.

In this paper an active vibration system is designed and built into the handheld probe. This paper describes the controller design then the design of the 

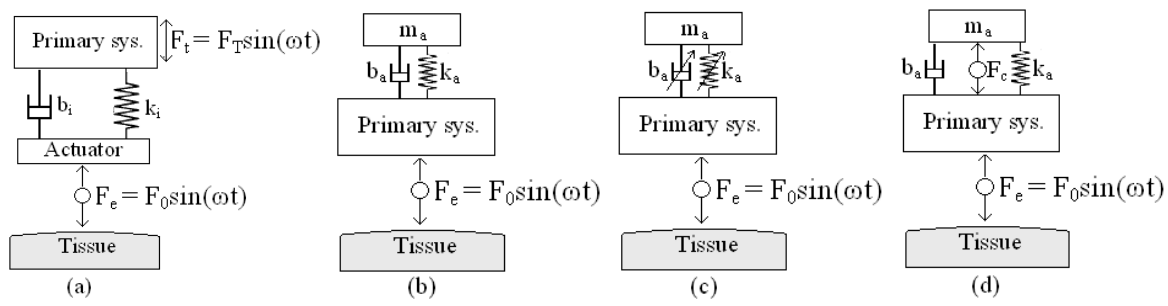

Fig. 1. Vibration reduction methods: (a) Vibration isolation (b) Passive vibration absorption (c) Semi-active vibration absorption (d) Active vibration absorption

overall device. Parameter identification of the absorber properties are then performed to optimize performance. Simulations and experiments are then performed to test the level of vibration absorption and the ability to create images with VE. But first, simulations are used to demonstrate the level of errors in VE that occur without vibration absorption.

\section{The Effect of Probe Vibration}

Like VE, the tissue is modelled as an $n$ DOF mass-spring-damper model. The tissue is vibrated from one side with the amplitude of $1 \mathrm{~mm}$ and the position of each mass is calculated at intervals in time. If the probe is stationary, the positions measured from the ultrasound images should be the same as the positions calculated from the simulation. To simulate probe vibration, a portion of the vibration displacement source is added to the simulation results and considered as measurements. The parameters of mass, viscosity and stiffness are then identified from the measurements by the VE equations.

Figure 2 shows the relationship between the amplitude of probe vibration and the relative error in estimating the tissue properties. The error is not the same at all elements/nodes of the modelled tissue, so are averaged to produce a single error value at each amplitude. As shown, there is a significant error introduced by
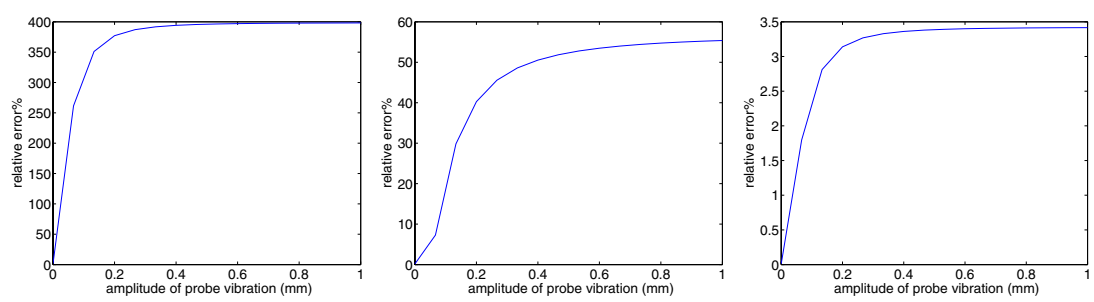

Fig. 2. Simulations of probe vibration on the error in VE. The left, middle and right plots corresponds to the error in the calculated mass, viscosity and stiffness respectively. The tissue is excited at $1 \mathrm{~mm}$ amplitude over a range of $10 \mathrm{~Hz}$. 
the probe vibration. For example, an absorption of $90 \%$ of the vibration results in a drop in the error in estimating viscosity from approximately $60 \%$ to $20 \%$.

\section{Controller Design}

The vibration absorber on the probe should be designed to minimize the vibration without requiring a large number of sensors (to minimize costs). A PI controller is chosen here with measurement of only the absorber mass acceleration. The control force of a PI controller with acceleration feedback is

$$
F_{c}=K_{p} \ddot{x}_{a}+K_{i} \dot{x}_{a}
$$

where $K_{p}$ and $K_{i}$ are the proportional and integral gain coefficients respectively and $x_{a}$ is the position of the absorber mass. Having this force as the control command, the equation of motion of the absorber mass is

$$
m_{a} \ddot{x}_{a}+c_{a} \dot{x}_{a}+k_{a} x_{a}=K_{p} \ddot{x}_{a}+K_{i} \dot{x}_{a}
$$

Taking the Laplace transform one has $m_{a} s^{2}+c_{a} s+k=K_{p} s^{2}+K_{i} s$. By selecting the $K_{p}$ and $K_{i}$ such that the roots of the above equation are placed on the imaginary axis at $\pm j \omega$, the absorber system will mimic a resonator at the frequency of $\omega$. This system can cancel the vibrations of the primary system at the frequency $\omega$. Solving the equation with $s= \pm j \omega$, one has

$$
K_{p}=m_{a}-k_{a} / \omega^{2}, \quad K_{i}=c_{a}
$$

With an electromagnetic actuator, the control command is the applied voltage. Therefore, the dynamics of the electromagnetic actuator must be considered. The block diagram representation of the absorber system with the electromagnetic actuator is provided in Figure 3. In this figure, $\mathrm{L}$ and $\mathrm{R}$ are the inductance and the DC resistance and $K_{f}$ and $K_{b}$ are the force sensitivity and back emf constants of the actuator. To get the velocity data from the acceleration data, a first order Butterworth highpass filter with $0.5 \mathrm{~Hz}$ cutoff frequency is used before integration to eliminate the low frequency drift of the accelerometer data.

Again, to have a vibration absorber at the frequency $\omega, K_{p}$ and $K_{i}$ should be selected such that the roots of the closed loop system are at $\pm j \omega$. Finding the closed loop transfer function of the whole system and forcing it to have poles at $\pm j \omega$, the integrator and proportional gains can be found

$$
\begin{gathered}
K_{p}=\frac{R\left(k_{a} / \omega^{2}-m_{a}\right)-L c_{a}}{K_{f}} \\
K_{i}=\frac{L\left(k_{a}-m_{a} \omega^{2}\right)+R c_{a}+K_{f} K_{b}}{K_{f}}
\end{gathered}
$$

It is straightforward to show that the highpass filter has a very small effect on $K_{p}$ and $K_{i}$ and therefore is neglected in (4) and (5). 


\section{Hand-Held Probe Design}

Figure 3 shows the hand-held device. Parts labelled as (c) and (d) are the primary actuator's coil and magnet. This actuator vibrates the tissue, while the ultrasound probe (a) images the tissue. The absorber actuator is mounted in an opposite way: its coil (e) is fixed and the magnet (f) moves. Therefore the absorber mass is simply the magnet of the actuator. This choice obviates an additional absorber mass, making the hand-held device lighter. Part (g) is one of the two absorber springs. There is no damper in the absorber; the inevitable coulomb friction between the magnet and the housing and also the rods and the bushings play the role of a damper. Parts (b) and (h) are the linear potentiometer and piezoelectric accelerometer respectively. The accelerometer is mounted on the absorber mass (the magnet) and provides the only feedback for the vibration absorption algorithm. The potentiometer signal is only used to operate the first actuator in closed loop for displacement control. Here a conventional PID controller is used and since the mathematical model of the vibrator is unknown, the coefficients of the PID controller are obtained by the Ziegler-Nicholas method [4].
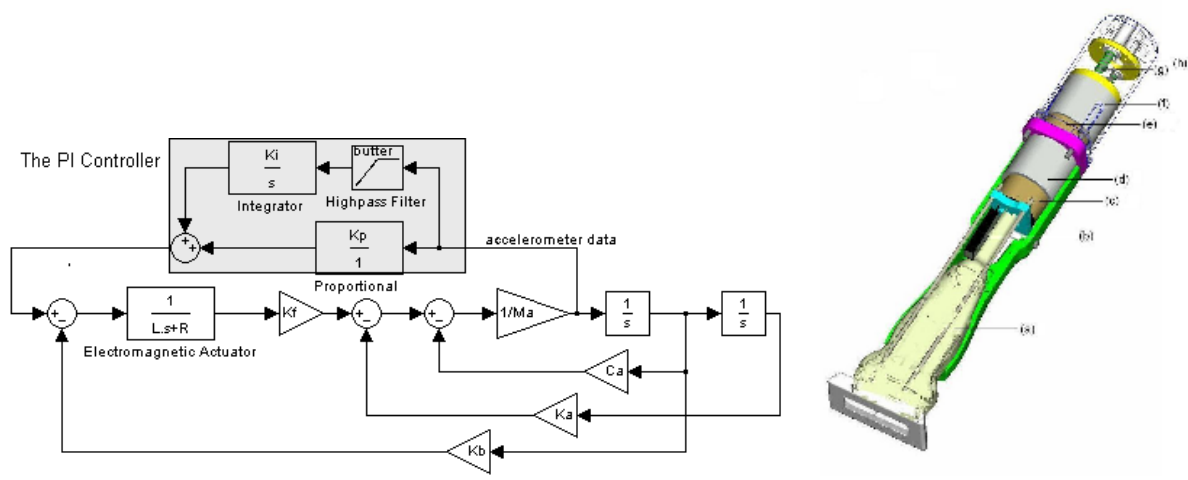

Fig. 3. Left: Controller for vibration absorber. Right: Exploded view of the hand-held probe. (a) ultrasound transducer; (b) potentiometer; (c)and (e) actuator coils; (d) and (f) actuator magnets; (g) absorber spring; (h) accelerometer.

The probe is designed for a desired frequency range of $10-50 \mathrm{~Hz}$ to cover a sufficient bandwidth of the tissue response. The values of $m_{a}, k_{a}$ and $b_{a}$ should be chosen from the stability analysis of the absorber. To cover the desired bandwidth, $m_{a}$ is 180 grams and $k_{a}$ is $650 \mathrm{~N} / \mathrm{m}$ (implemented as two serried Spaenaur B-646 springs). The value for $c_{a}$ can vary between $1 \mathrm{~kg} / \mathrm{s}$ and $15 \mathrm{~kg} / \mathrm{s}$ since damping comes from friction.

\section{Identification of the Absorber Parameters}

After manufacturing the device, the exact values of $m_{a}, k_{a}$ and $b_{a}$ need to be measured. The value of $m_{a}$ was measured by a scale and is 199 grams. To mea- 
sure $k_{a}$ and $b_{a}$, a free vibration test on the absorber mass was done and the acceleration measured by the accelerometer. Assuming that the free vibration is of the form $x(t)=A e^{-\zeta \omega_{n} t} \sin \left(\omega_{d} t+\phi\right)$ where $\omega_{n}=\sqrt{(k / m)}$ is the natural frequency and $\zeta$ is the damping coefficient and $\omega_{d}$ is the frequency of damped oscillation, the two unknowns $\omega_{n}$ and $\zeta$ can be found by matching the measured motion to the free vibration equation. This gives $\omega_{n}=9.0 \mathrm{~Hz}$ and $\zeta=0.5$. These translate into $k_{a}=636.4 \mathrm{~N} / \mathrm{m}, c_{a d}=11 \mathrm{Kg} / \mathrm{s}$, and $\omega_{d}=7.8 \mathrm{~Hz}$. The coulomb friction is the major source of damping (plus eddy currents induced by the moving magnet in the housing) and the given $c_{a}$ value is the result of linearization. Because of its nonlinearity, the measured $c_{a}$ is valid for the frequency of the test so is labelled $c_{a d}$. The $c_{a}$ at the frequency $\omega$ is then modelled as 7 ]

$$
c_{a}=\frac{\omega_{d}}{\omega} c_{a d}
$$

\section{Simulation Results}

The performance of the proposed active vibration absorber is investigated by simulation. Figure 4 compares the vibration of the probe with and without active absorption. An excitation is applied to the probe held by a simple model of a human arm (stiffness of $100 \mathrm{~N} / \mathrm{m}$ and damping of $2 \mathrm{~kg} / \mathrm{s}$ ). An attenuation level of $70 \mathrm{~dB}$ is achieved with the active vibration absorber after $5 \mathrm{sec}$.
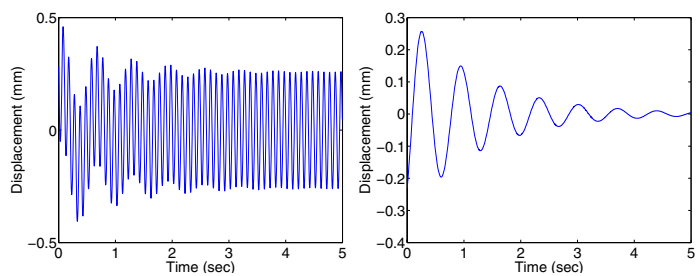

Fig. 4. Simulation of probe motion without and with the vibration absorber (left and right respectively). Tissue is excited at $10 \mathrm{~Hz}$ with an amplitude of $1 \mathrm{~mm}$.

\section{$7 \quad$ Experimental Results}

Several experiments were carried out on the probe to show the effectiveness of the active vibration absorber. A mechanical arm was built to hold the probe in order to perform repeatable and comparable experiments. The arm has a stiffness of $140 \mathrm{~N} / \mathrm{m}$, mimicking the stiffness of the human arm holding the probe. Speckle tracking is performed by a window-based cross-correlation algorithm [5]. An OPTOTRAK 3020 (NDI, Waterloo, Canada) tracker with a measurement rate of $400 \mathrm{~Hz}$ is used to track the motion of the probe. The OPTOTRAK is only used to investigate the performance of the vibration absorber and is not used in the vibration absorption control. In the first experiment, the probe vibrates a 

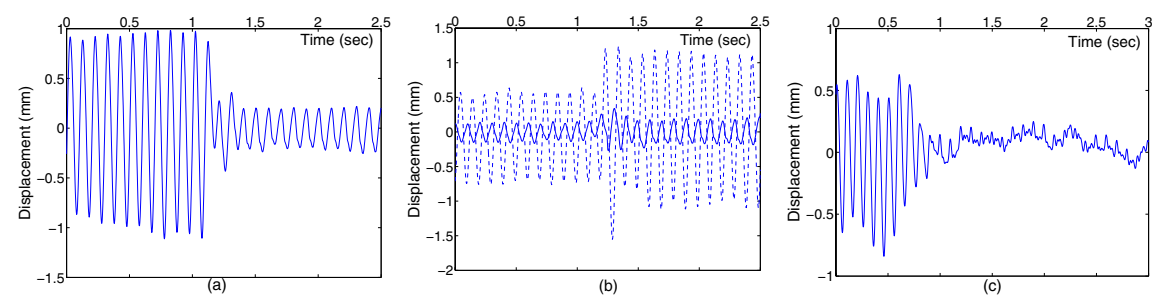

Fig. 5. Experimental results. (a) Probe vibration on a gelatin phantom with a mechanical arm, before and after activating the vibration absorption ( $\mathrm{t}=1.1 \mathrm{~s})$. (b) Another test looks at an increase in the excitation amplitude from $2 \mathrm{~mm}$ to $3 \mathrm{~mm}$ at $\mathrm{t}=1.3$ s. Dashed and solid curves show the motion of the probe without and with the active vibration absorber respectively. (c) Probe vibration for experiments with a human operator on real tissue. The vibration absorber is turned on at $\mathrm{t}=0.7 \mathrm{~s}$.
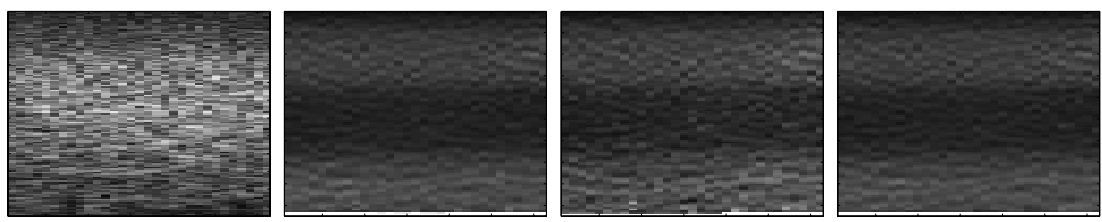

Fig. 6. left to right respectively: B-mode image, best-case example of static elastogram, worst-case static elastogram, and the VE of a three layered phantom

phantom at a frequency of $10 \mathrm{~Hz}$. Figure 5 a shows that the amplitude of probe vibration is reduced from $0.9 \mathrm{~mm}$ to $0.2 \mathrm{~mm}$ when the active vibration absorber is turned on at $t=1.1 \mathrm{~s}$. To see the performance of the probe to absorb forces with variable amplitude, two experiments with and without active vibration absorption are done. In these experiments, the excitation amplitude is increased from $2 \mathrm{~mm}$ to $3 \mathrm{~mm}$ at $\mathrm{t}=1.3 \mathrm{~s}$ (Figure $5 \mathrm{~b}$ ). The amplitude of vibration is increased from $0.6 \mathrm{~mm}$ to $1.1 \mathrm{~mm}$ without vibration absorption and from 0.12 $\mathrm{mm}$ to $0.17 \mathrm{~mm}$ with the vibration absorption. The experiment shows an increase in the attenuation level when the excitation force is increased. In Figure 5c, the probe is held by a human operator on the forearm of a human subject. In this experiment, the vibration absorber decreases the peak to peak motion from 1.28 $\mathrm{mm}$ to $0.13 \mathrm{~mm}$. Because the probe is held by hand and applies a slightly uneven pressure to the tissue, a net motion at a low $2 \mathrm{~Hz}$ frequency (compared to the $10 \mathrm{~Hz}$ vibration frequency) is seen. This motion is easily seen in the frequency domain and excluded from VE. Experiments with a $20 \mathrm{~Hz}$ excitation frequency show similar $0.9 \mathrm{~mm}$ to $0.2 \mathrm{~mm}$ vibration reduction. A B-mode ultrasound image and three real elastograms obtained from a three layered gelatin phantom are shown in Figure 6. The middle layer of the phantom is stiffer than the other layers but exhibits the same acoustic properties. The stiff layer is not visible in the B-mode image but it is detectable in the elastograms. The static elastograms (second and third images) are obtained by applying a 0.01 compression to the 
phantom slowly and rapidly acquiring RF data during the compression, to ensure high correlation coefficients between consecutive echo frames. A vibro-elastogram is obtained from the same phantom by vibrating it with a $5 \mathrm{~Hz}$ band limited white noise. Static elastograms can be noisy (e.g. third image in Figure 6), but vibro-elastograms are more robust to noise due to their averaging step performed in frequency domain.

\section{Conclusions}

Despite the uncertainties in the absorber model and the friction model, the vibration absorber performed well. The control law of the active vibration absorber requires only the measurement of the absorber mass acceleration. Also, the control law is decoupled from the primary system and the amplitude of the excitation force. This property is inevitable for the probe because of two main reasons. First, the properties of the primary system can change dramatically since the probe can be held by various operators with various inertia, damping and stiffness properties. Second, the probe vibrates various types of tissues with various mechanical properties so the excitation force can vary considerably. As a final note, the designed PI controller has the advantage of not requiring a double integration from the acceleration data compared to the PD controller proposed in [1. Since the accelerometer data has a low frequency variable drift, it is impossible to twice integrate the acceleration data because of the accumulation of error. By replacing the bushings with linear bearings and increasing the air gap between the magnet and the housing to decrease the eddy currents, the performance of the vibration absorber will be improved to the simulation results. Given a working probe, work is now underway on clinical applications of VE.

\section{References}

1. Elmali, H., Renzulli, M., Olgac, N.: Experimental comparison of delayed resonator and PD controlled vibration absorbers using electromagnetic actuators: J. of Dynamic Systems, Measurement and Control. Vol. 122 (2000) 514-520.

2. Jalili, N.: A comparative study and analysis of semi-active vibration-control systems. J. of Vibration and Acoustics, Trans. of the ASME, Vol. 124(4) (2002) 593-605.

3. Korenev, B.G., Reznikov, L.M.: Dynamic vibration absorbers: theory and technical applications. John Wiley, New York (1993).

4. Ogata, K.: Modern control engineering. 4th edn. Prentice Hall, New Jersey (2002).

5. Ophir, J., Alam, S.K., Garra, et al.: Elastography: imaging the elastic properties of soft tissues with ultrasound. J Med Ultrasound, Vol. 29 (2002) 155-171.

6. Sun, J.Q., Jolly, M.R., Norris, M.A.: passive, adaptive and active tuned vibration absorbers-a survey. J of Vibration and Acoustics Vol. 117 (1995) 134-242

7. Thomson, W.T., Dahleh, M.D.: Theory of vibration with applications. 5th edn. Prentice Hall, New Jersey (1998)

8. Turgay, E., MacIntosh, S., Rohling, R., Salcudean S. Parameter identification of tissue lumped models based on sequences of ultrasonic strain images. Second Intl. Conf. on Ultrasonic Measurement and Imaging of Tissue Elasticity. Corpus Christi, Texas (2003) 29. 